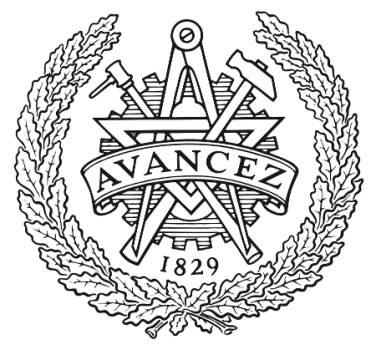

CHALMERS

UNIVERSITY OF TECHNOLOGY

\title{
Comparing a Humanoid Tutor to a Human Tutor Delivering an Instructional Task to Children
}

Downloaded from: https://research.chalmers.se, 2023-04-26 01:25 UTC

Citation for the original published paper (version of record):

Serholt, S., Basedow, C., Barendregt, W. et al (2015). Comparing a Humanoid Tutor to a Human

Tutor Delivering an Instructional Task to Children. 2014 IEEE-RAS International Conference on Humanoid Robots: 1134-1141. http://dx.doi.org/10.1109/HUMANOIDS.2014.7041511

N.B. When citing this work, cite the original published paper. 


\title{
Comparing a Humanoid Tutor to a Human Tutor Delivering an Instructional Task to Children
}

\author{
Sofia Serholt ${ }^{1}$, Christina Anne Basedow ${ }^{2}$, Wolmet Barendregt ${ }^{1}$, and Mohammad Obaid ${ }^{3}$ \\ ${ }^{1}$ Department of Applied IT, University of Gothenburg, Gothenburg, Sweden \\ ${ }^{2}$ School of Humanities \& Social Sciences, Jacobs University, Germany \\ ${ }^{3}$ t2i Lab, Chalmers University of Technology, Gothenburg, Sweden \\ sofia.serholteait.gu.se
}

\begin{abstract}
This paper presents a study that compares a humanoid robotic tutor to a human tutor when instructing school children to build a LEGO house. A total of 27 students, between the ages of 11-15, divided into two groups, participated in the study and data were collected to investigate the participants' success rate, requests for help, engagement, and attitude change toward robots following the experiment. The results reveal that both groups are equally successful in executing the task. However, students ask the human tutor more often for help, while students working with the robotic tutor are more eager to perform well on the task. Finally, all students get a more positive attitude toward a robotic tutor following the experiment, but those in the robot condition change their attitude somewhat more for certain questions, illustrating the importance of real interaction experiences prior to eliciting students' attitudes toward robots. The paper concludes that students do follow instructions from a robotic tutor but that more long-term interaction is necessary to study lasting effects.
\end{abstract}

\section{INTRODUCTION}

In the field of educational robotics, there have been numerous research endeavors which aim to explore possible applications and functions of robots used for social interaction or learning within educational settings, such as [5],[18] and [13]. Such robots can take the role of tutors, peers or tools within learning environments. Using robots as tools or 'digital manipulatives' in the teaching of concepts within e.g. science, technology or engineering has been exercised for some time [3], however the effects of equipping robots with tutoring functions remains relatively unexplored in real classroom settings.

Previous research has suggested that robots are preferred over virtual agents by users in terms of social interaction [2], performance [14], and enjoyment [20]. However, such studies do not reveal how a humanoid robot compares to common classroom practice, where a tutoring role is assumed by a human teacher. Since a robotic embodiment allows for a physical interaction that is not possible with a virtual agent, we aim to explore whether students will behave similarly or differently toward a tutoring humanoid robot in comparison to a tutoring human.

In the case of a humanoid robot tutor, the tutoring situation implies partly that students have to accept delivered instructions. This notion is the main motivation for this study in which we investigate how students respond to instructions delivered by a robot. Thus, in this paper we present a comparative experiment that took place in a classroom setting where students were requested to complete an instructional task conveyed either by a humanoid robot tutor (RT) or a human tutor (HT).

This paper is outlined as follows: The next section presents related work within the HRI field. Thereafter, we present our hypotheses as related to the literature, followed by presentations of the experimental setup, apparatus, the subjective and objective measures collected, as well as a description of the experimental procedure. The next chapter comprises the results of the experiment as well as discussions in relation to each hypothesis. Finally, our concluding remarks along with some suggestions for future work are presented.

\section{RELATED WORK}

There is a body of HRI research investigating assistive robotics in different domains such as health [9], consumption [17], and education [18] where most assistive robots act as a mediated interface that can aid the humans with their tasks.

Educational robot-aided learning has partly emerged from the significant work devoted to exploring how virtual agents may enrich learning experiences within virtual learning environments. Lifelike virtual characters or animated pedagogical agents displayed within such environments offer the possibility of engaging and motivating students within a learning task through verbal and nonverbal communication [7]. Studies have shown that animated pedagogical agents contribute to students' learning experiences in terms of achievement scores, attitudes, retention of learning [28], and study outcomes [26]. Research has furthermore demonstrated the key role that different levels of embodiment play in users' perceptions of artificial entities. The level of embodiment may range from a static or animated image of a character on a computer screen to a physically present robot [15].

Mubin et al. [18] presented an overview on research and development of robots used in education. They pointed out that one of the main categories for the role of an educational robot is a tutor. Several researchers have investigated the use of robots in education, for example, Han et. al. [6] studied the potential of using home robots for children to learn English. They compared Home Robot-Assisted Learning (HRL) with other instructional media: books/audiotapes and web-based instructions. Their findings revealed that HRL did promote 
and improve children's concentration, interest, and learning achievement.

Further, Saerbeck et. al. [22] evaluated the effect of social supportive behavior of a robot mediated interface, and their results revealed an increase of students' learning efficiency. In addition, Shin and Kim [24] interviewed 85 students (from three school levels with an average age of 14 years) to investigate their perceptions and attitudes toward robots in education. Some of their results revealed that the students agreed that they could learn from a robot but they did not think it would act as a teacher. This was mainly due to the lack of expressive behaviors in robots, which can be expected from human teachers. The aforementioned research indicates promise in the field of HRI where robots can act as tutors for students, however, comparisons should perhaps also be made to human tutors in order to discern how interaction with robots differs from a more conventional tutoring situation.

Cormier et al. [4] investigated differences in participants' (adults) responses toward a robot versus a human experimenter in an obedience scenario. Their results revealed that the human condition was more authoritative, however, an authoritative robot could still pressure participants to complete tedious tasks against their will. These results suggest that we can expect certain compliance with instructions in an experimental situation even when those instructions are delivered by a robot. Even so, it does not reveal whether children would also potentially comply with meticulous instructions coming from a robot.

Moreover, Kim et al. [11] conducted a study comparing participants' perceptions of potential communicative constraints in interaction with either a social humanoid robot or a human in an envisioned tutoring scenario. They conducted a survey study with 230 higher education students based on their theoretical framework with three socialoriented communication constraint parameters: feelings, nonimposition, and disapproval; as well as two task-oriented parameters: clarity and effectiveness. Their results indicate that participants would be more concerned about socialoriented issues when interacting with humans, in that they were significantly more concerned with avoiding hurting the human feelings, inconveniencing the human, as well as avoiding being disliked by the human. The task-oriented parameters, on the other hand, were valued to be equally important in both cases.

Although the results from Shin and Kim [24] are relevant for our target age group, and the study by Kim et al. [11] relates to a human-robot comparison, these are both based on interviews or survey results where no real interaction with humanoid robots was conducted. In this context, responses may be related to participants' preconceptions acquired through media, rather than personal interaction experience.

In this study, we compare an HT and a humanoid RT instructing children to build a LEGO house as shown in Fig. 1. To our knowledge, a comparative study of a human versus a humanoid robot tutor with children in a classroom has not previously been investigated.

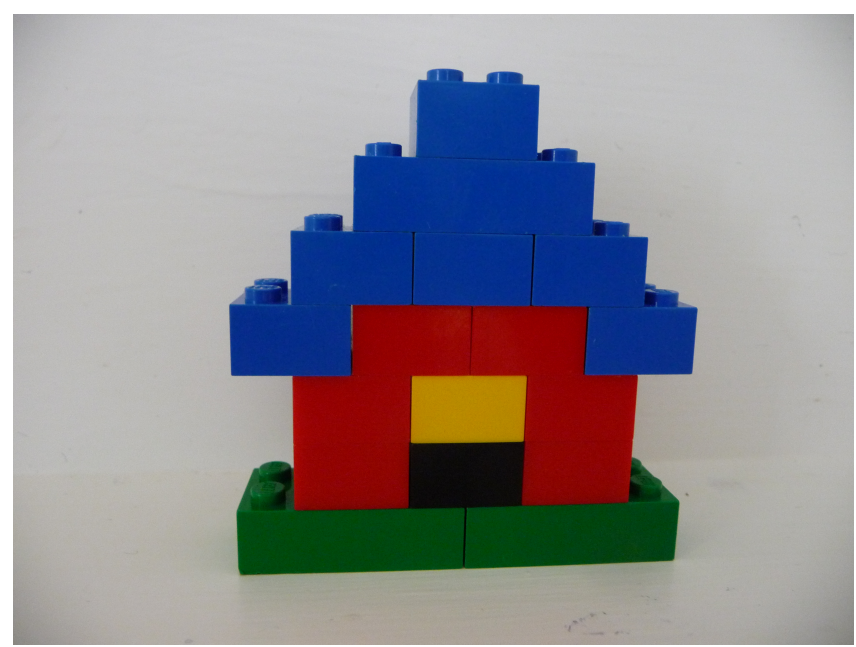

Figure 1: The final outcome of a correct LEGO house

\section{EXPERIMENT}

The main objective of this experiment is to explore the extent to which a child can follow basic instructions from a humanoid RT, and to examine the similarities and differences in engagement levels between human-human interaction and human-robot interaction within a simply formatted, instruction based activity. Another aim is to investigate how this interaction with an RT influences the students' attitudes toward it. The interaction was defined as the cooperation between student and tutor and the variation of verbal and non-verbal communication that was exchanged during the activity. This experiment explored to what extent interaction can be expected and can develop between child and robot in a single, short interaction, and what the differences are between an HT and an RT in this type of interaction. The simple instruction based activity consisted of thirteen verbal instructions, in which participants were given directions to build a house out of LEGO bricks.

The hypotheses of the experiment are: (H1) we expect that the HT condition will lead to increased student attention and success, measured through correct LEGO house completion and the number of requests for a repetition of the instruction. Comparative studies on human-human and human-robot interaction indicate higher levels of engagement with human experimenters in comparison to embodied robot experimenters, even when delivering the exact same instructions [10][12].

(H2) Furthermore, students will be more inclined to ask for help from the HT than from the RT. This is anticipated due to higher levels of student experience with human teachers/tutors versus robotic tutors. In addition, the ease and inherent component of the interaction process with the HT in comparison to the RT [25] will lead to a more natural interaction with the HT and greater requests for help [12].

(H3) We also expect that students will be less engaged with the RT than with the HT, measured through gaze attention/ direction and post-engagement questionnaires. Research indicates that engagement and face tracking with human 


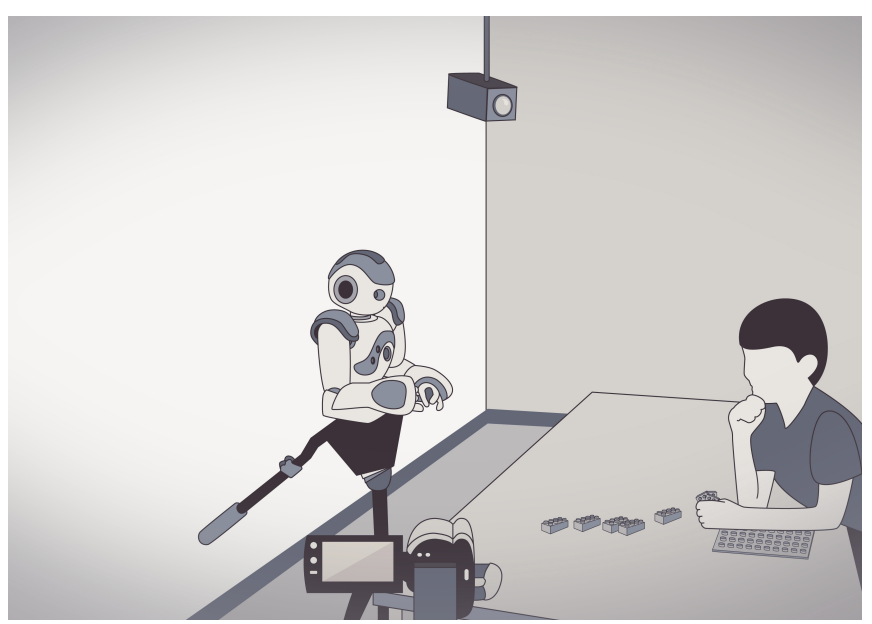

(a)

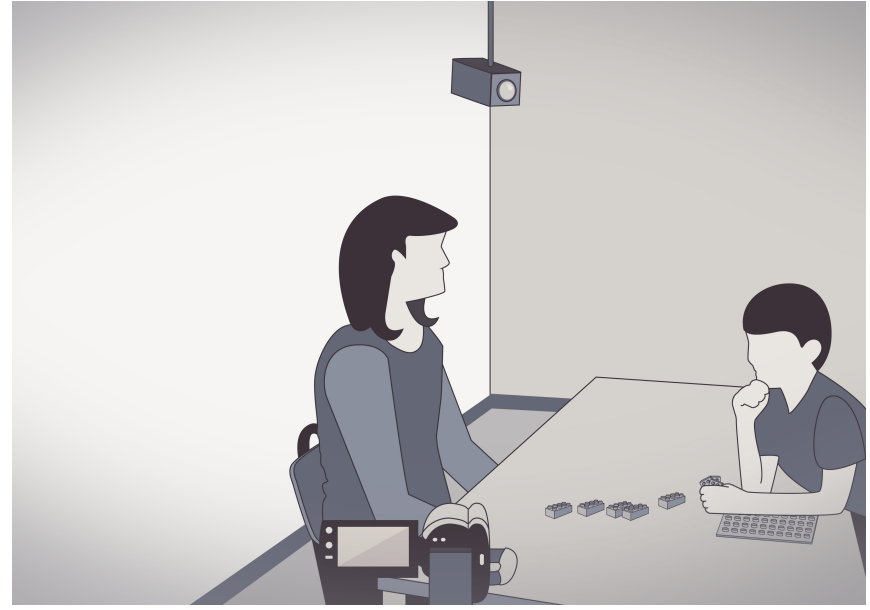

(b)

Figure 2: An illustration of the experimental setups with a human participant. Figure (a) illustrates the setup for a robot instructor, while figure (b) illustrates the setup for a human instructor

inter-actors is higher than with robot inter-actors [12][25]. Eye gaze is a central component of interaction, turn-taking and engagement in both verbal and non-verbal communication, and we anticipate higher levels of engagement with the HT, based on engagement patterns in conversation, often measured through eye gaze (direction, mutual gaze[8][25]).

(H4) Finally, we expect the student's interaction with the RT to result in a more positive attitude toward robots, measured as a decrease of their subjective scores on the childfriendly Negative Attitudes toward Robots Scale (NARS). Studies that examine the use of robotics in supporting education have indicated that students accept robots as interaction partners, and after initial interaction, typically have a strong desire to continue engaging with the robot [27].

\section{A. Experimental Setup}

We focus on investigating human-robot interaction in a classroom setting. In this work, we compare students' success when requested to complete an instructional task conveyed either by a humanoid robot or a human tutor. Thus, in this experiment, we used a between-subject design with two experimental conditions: (1) a human tutor and (2) a humanoid robot tutor.

For the purpose of our study, we engaged 27 school children (15 males and 12 females), between 11 and 15 years old (mean=13, SD=1.4) to participate. One of the male participants was absent on the day of the experiment, and one female participant was omitted from analysis due to technical problems with the robot, leaving 25 students (14 males and 11 females) in total. The first condition (HT) comprised 13 participants ( 8 males and 5 females), and the second condition (RT) comprised 12 participants (6 males and 6 females). All participants came from two classes in a Swedish secondary school.

The following sections describe the details of the experiment's apparatus, measurements obtained and procedure.
1) Apparatus: The study was arranged in a separate classroom at the participating students' school. As illustrated in Fig. 2, the room was equipped with a chair, a table, and three cameras. On the table, there was a pile of LEGO pieces of various shapes and colors that participants could interact with to fulfill the task of building a LEGO house according to the instructions. This task was designed based on a task for following oral instructions, where step-by-step audio instructions led to the drawing of a snowman. However, since we did not want to test children's artistic talents LEGO was the material of choice. Many children have previously been exposed to LEGO, and the varied blocks (both in size and color) allow for instructions to be formulated objectively for this task. Only 15 bricks from the pile of bricks were required to successfully complete the house. The additional LEGO pieces were added in order to entice the student to build something other than what was instructed and to make the task less obvious.

In the first condition, the instructor was a human teacher who would sit on the opposite side of the table facing the participant as illustrated in Fig. 2a. In the second condition, a humanoid robot instructor would face the student and deliver the instructions as illustrated in Fig. 2b. A Wizardof-Oz method was employed where the wizard, seated in an adjacent room, controlled the robot by initiating each instruction at appropriate times through a software interface. The robot tutor was able to track the student's face and perform idle movements (head movements, arm movements, and yawns) to increase believability of the humanoid robotic tutor as an autonomous actor.

In this setup, we used the Nao T14 torso robot from Aldebaran Robotics ${ }^{1}$. The robot was fixed on a tripod situated at the same height as the edge of the table as illustrated in Fig. 2a. The third camera, not visible in the illustration, was a small web-camera placed directly below the robot tutor

${ }^{1}$ http://www.aldebaran.com 
on the tripod stand or to the left of the human tutor. The instructions in the RT condition were prerecorded sentences with the transformed robot-like voice of the HT. The decision to use a transformed version of the HT voice was based on the fact that we did not want children to perform worse in the robot condition because of a misunderstanding of Textto-Speech generated instructions. This robot-like voice, along with other versions, had been pre-tested with several children to ensure understandability as well as naturalness. These audio files were then individually initiated by the wizard through a simple custom made graphical interface for instant audio playback.

2) Subjective Measures: The subjective measures consisted of two questionnaires: (1) the Negative Attitudes toward Robots Scale (NARS), based on the work by Nomura and Kanda [19] who identified a set of sub-scales relating to people's negative attitudes toward robots, and (2) a Task and Tutor Engagement Questionnaire [16].

NARS is a Likert-scale questionnaire which was originally developed for use by adults to explain differences in behavior between participants during their interactions with a robot. A higher NARS score means a more negative attitude toward robots. It has not been used to determine children's attitudes toward robots. Each question of the NARS relates to one of three following sub-scales: Negative Attitudes toward (S1) Situations and Interactions with Robots, (S2) Social Influence of Robots, and (S3) Emotions in Interaction with Robots. In order to adapt this questionnaire to be used by children, all authors discussed each of the questions to clarify their intention and adapt the language and context to school-aged children (10-15 years old). The questions were thereafter translated to Swedish and piloted with a 13-year old girl in order to discover potential comprehension issues, followed my minor alterations to some wordings. The final questions used are presented in Table I.

The Task and Tutor Engagement Questionnaire was created to specifically determine how engaging the activity and interaction was perceived to be from the perspective of the student. The task engagement questions, highlighting both affective and cognitive engagement were designed based on the research by [16] and contained 14 Likert scale questions ( $1=$ completely disagree to $5=$ completely agree). The questions used are presented in Table II.

The questionnaire was given to participants after the task was completed and it intended to measure their immediate reaction to the task [16]. Social engagement questions gauged the enjoyment, depth, breadth and level of interaction with the instructor, based on the research of [21]. Finally, questions also asked about the motivation to either assist the robot as an interaction partner and/or to continue socially engaging with the robot, examining at a minimal level the degree to which a socio-emotional bond had started to form between tutor and student. These questions were adapted from the scales developed by [23].
3) Objective Measures: For the analysis of the video data a coding scheme was developed to be used in ELAN ${ }^{2}$. The coding scheme consisted of the following codes: gaze, verbal behaviour, non-verbal behaviour and LEGO house building actions. For gaze we coded whether the student was gazing at the instructor (human or robot), at the LEGO pieces, at the camera, or elsewhere. For verbal behavior we identified the student stating: repeat, next, request help, or repeat several steps. Non-verbal behavior identified any non-verbal cues the student expressed, such as sounds, head shaking, or laughing (apart from gaze). Finally, LEGO house building actions were coded to identify whether the student was building the house correctly or incorrectly. The video analysis was completed by one primary coder, followed by an additional coder who checked the initial analysis.

Coding the LEGO house building instructions, spoken by the instructor, and subsequent student 'building' actions allowed for an understanding of how well the student followed basic directions in both conditions. It created an objective measure to explore the similarities and differences in 'correct' versus 'incorrect' house-building with an RT or HT. For each LEGO house-building instruction given to the student, we coded whether it was performed 'correctly' or 'incorrectly'. Once the student made a mistake, all subsequent instructions were coded as incorrect. At the end of the activity, the final independent measure was whether the student had built the complete house 'correctly' or 'incorrectly'.

The function of gaze (eye movement) has long been seen as a non-verbal social channel that expresses how we attend to varying stimuli, how engaging we find the stimuli and the amount of attention we are willing to assign to the stimuli [1]. The role of gaze as an attending behavior is a pertinent measure to understand the level of interest and engagement of the subject within an activity, the interaction with others and the desire to remain engaged or to disengage [25]. Gaze is a particularly pertinent measure within this study, as research indicates that an embodied tutor (such as the physically present NAO) is accepted and treated with human-like social interaction rules in comparison to nonembodied artificial entities [8]. Gaze of the student was thereby used as an objective measure to indicate whether the student was acknowledging the instructions, and whether the student would look at the human or robot tutor, particularly during turn-taking, which would indicate acceptance of the instructions as natural conversation [25].

4) Procedure: The experiment started with distribution of the child-friendly NARS questionnaire on paper one week prior to the experiment. Students were asked to fill out this questionnaire individually and return the form to their teacher.

During the first two days of the experiment only the HT condition was performed. During these days the students were randomly taken from each class in order to ensure equal distribution of age and gender across the two conditions. Dur-

\footnotetext{
${ }^{2}$ http://tla.mpi.nl/tools/tla-tools/elan/
} 
TABLE I: Questions in the child-friendly NARS (*inverse item)

\begin{tabular}{|l|r|c|}
\hline Nr & Question & Sub-scale \\
\hline 1 & I would feel uneasy if robots really had emotions. & S2 \\
\hline 2 & Something bad might happen if robots developed into living beings. & S2 \\
\hline 3 & I would feel relaxed talking with robots. ${ }^{*}$ & S3 \\
\hline 4 & I would feel uneasy if I had to do my school work with help of a robot. & S1 \\
\hline 5 & If robots had emotions I would be able to make friends with them.* & S3 \\
\hline 6 & I feel good being with robots that have emotions. ${ }^{*}$ & S3 \\
\hline 7 & The word "robot" means nothing to me. & S1 \\
\hline 8 & I would feel nervous using a robot in front of other people. & S1 \\
\hline 9 & I would hate the idea that robots or intelligent computers were making & S1 \\
\hline 10 & I would feel very nervous just standing in front of a robot. & S1 \\
\hline 11 & I feel that if I depend on robots too much, something bad might happen. & S2 \\
\hline 12 & I would feel paranoid talking with a robot. & S1 \\
\hline 13 & I am concerned that robots would be a bad influence on younger children. & S2 \\
\hline 14 & I feel that in the future society will be dominated by robots. & S2 \\
\hline
\end{tabular}

TABLE II: Questions of the Task and Tutor Engagement Questionnaire

\begin{tabular}{|l|l|l|l|}
\hline Nr & Task Engagement & Nr & Social Engagement \\
\hline Q1 & I enjoyed this activity & Q8 & I would like to do another activity with the instructor \\
\hline Q2 & I found this activity hard & Q9 & I was worried about doing a good job for the instructor \\
\hline Q3 & I would like to continue with this activity & Q10 & I wanted to keep building with the instructor \\
\hline Q4 & It was important for me to do a good job & Q11 & I felt like the instructor and I were part of the same team \\
\hline Q5 & I found this activity easy to understand & Q12 & I found the instructor helpful \\
\hline Q6 & I have done activities like this before & Q13 & I found it easy to follow instructions presented to me \\
\hline Q7 & I found this activity boring & Q14 & I found the instructor boring \\
\hline
\end{tabular}

ing the final and third day the RT condition was performed with the remaining students.

All students were informed about their right to withdraw their participation during and after the experiment. They were also asked not to discuss their experiences with their classmates until the end of the third day.

For both conditions the tutor, either the human or the robot, introduced the activity by explaining that they would be building a LEGO house, based on instructions conveyed by the tutor. The tutor also explained that she would not be able to help the student (to allow for some congruence between conditions) but that the instruction could be repeated if the student said "Repeat". Thereafter the 13 instructions to build the house were given, starting from two green pieces of LEGO already being placed on a LEGO plate. An example of such an instruction is the following: "Place 2 red pieces with 4 dots on each side of the black piece". Following all instructions correctly would lead to the house depicted in Fig. 1.

Both the RT and the HT could make use of some standard transitions between the instructions and prompts to continue working on the task. After the last instruction the tutor thanked the student for participating. Immediately following the task, the post-questionnaire comprising the NARS and Task and Tutor Engagement Questionnaire was administered.

\section{Results And Discussion}

\section{A. Hypothesis (H1): Success}

In order to compare the student success, we used two measures: the percentage of students building the complete house correctly, and the number of times the students requested a repetition of an instruction. We hypothesized that students would have a higher success rate in the HT condition, resulting in a higher percentage of students building the house correctly and a lower number of requests for repeats. However, the distributions in the two groups did not differ significantly for either building the house correctly (Mann-Whitney $\mathrm{U}=61.500 \mathrm{n}_{H T}=13, \mathrm{n}_{R T}=$ $12, \mathrm{p}>0.05$ two-tailed) or for asking to repeat instructions (Mann-Whitney $\mathrm{U}=67.000 \mathrm{n}_{H T}=13, \mathrm{n}_{R T}=12, \mathrm{p}>0.05$ two-tailed). Although the success rate in house completion was similar for both conditions, it is possible that some instructions lead to more problems in one of the conditions. Further analysis of the instructions leading to an incorrect house revealed that students experienced problems with instructions 5 and 7, regardless of the condition. This suggests that there were task-related issues with the formulation of the instructions, rather than any connection to the human-robot comparison. It could also be the case that different students perceive these types of instructions in different ways, or have different levels of familiarity with LEGO.

\section{B. Hypothesis (H2): Requesting Help}

Students in the HT condition asked for help on average 1.69 times while students in the RT condition never asked for help. The distributions in the two groups differed significantly (Mann-Whitney $\mathrm{U}=18.000 \mathrm{n}_{H T}=13, \mathrm{n}_{R T}=12$, $\mathrm{p}<0.001$ two-tailed). This is likely due to the amount of time students have spent interacting with human tutors/teachers. The inherent nature of this interaction, and the natural tendency to ask for help from a human tutor or teacher explains our result. Furthermore, the RT condition only had minimal engagement gestures which can lead to interaction seeming 'artificial', in comparison to the HT condition which 
automatically would have displayed more engagement gestures. It could be inferred that the more 'natural' interaction with the HT increases the student's comfort in asking for help. In addition, the student could infer that the RT could not truly 'hear' or 'help' them due to prior experience with other technologies which lack social interactions, further reducing the likelihood to ask for help. This leads to a discussion on implications for further research.

The significant difference in students asking for help cannot be correlated with differences in successful LEGO house completion. This opens a discussion on whether a humanoid robotic tutor (even with limited engagement behaviors) allows for students, working on an independent task, to increase the amount of time they explore the activity without asking for help. It should certainly be explored outside a controlled experimental setting whether having a robot tutor, in some activities, encourages more critical thinking and problem solving on behalf of the student as they become aware they cannot ask for meaningful help, or whether this causes them to give up on the activity altogether. Similarly, another interesting exploration is whether students' hesitancy toward seeking help from a robot tutor will be affected by robots being equipped with improved speech recognition in the future, affording the possibility of robots providing useful help upon request.

\section{Hypothesis (H3): Engagement}

The percentage of time the students gazed at the instructor, the LEGO pieces, the camera, and elsewhere is shown in Figure 3. We hypothesised that students' engagement as measured by the time they gazed at the instructor and the LEGO blocks instead of at the camera and somewhere else would be higher for students in the HT condition. The times gazed at the camera in the HT and RT group were $0.92 \mathrm{~s}$ and $8.92 \mathrm{~s}$ respectively; the distributions in the two groups differed significantly (Mann-Whitney $\mathrm{U}=2.500 \mathrm{n}_{H T}=$ $13, \mathrm{n}_{R T}=12, \mathrm{p}<0.001$ two-tailed). This indicates that the presence of cameras was an influencing factor in this set-up. It could perhaps be indicative of attempts to establish contact with someone besides the robot, e.g. due to an understanding of being observed by the Wizard and/or the researcher.

Furthermore, the times gazed at the LEGO blocks in the HT and RT group were 78.92s and 69.08s respectively; the distributions in the two groups differed significantly (Mann-Whitney $\mathrm{U}=32.500 \mathrm{n}_{H T}=13, \mathrm{n}_{R T}=12, \mathrm{p}<0.05$ two-tailed) which most likely suggests task-engagement.

The average answers to each of the questions in the Task \& Tutor Engagement Questionnaire are provided in Fig. 4.

The distributions in the two groups differed only significantly for one engagement question: students in the RT condition answered more affirmatively that it was important for them to perform well on this task (Q4) (Mann-Whitney $\mathrm{U}=43.500 \mathrm{n}_{H T}=13, \mathrm{n}_{R T}=12, \mathrm{p}<0.05$ two-tailed). The novelty of interacting with robots could be a factor here, or it could simply be a matter of a desire to perform well within a research situation. If, however, this desire to perform well is somehow related to social-oriented communicative issues, as described by Kim et al. [11] in the sense that students desired to perform well in order to avoid either inconveniencing or being disliked by the robot, these results would stand in contradiction to the findings made by Kim et al. [11] with adult participants. Perhaps it could be the case that social-oriented communicative issues are in fact deemed important for people interacting with robots, but that this desire can only be realized through true robot interaction experiences. On the other hand, it could also be that the students in the HT condition felt less demand to perform well due to feelings of security invoked by the HT. Or, as discussed above, children may have relied more heavily on their own abilities in the robot condition (limited access to assistance) and completing the activity successfully became more important. Further studies thus need to be performed in order to understand whether students will still be as eager to perform well on a task guided by a robot once the potential novelty effect has worn off.

\section{Hypothesis (H4): Attitudes}

We hypothesised that students' attitudes toward robots would be influenced positively by their experience with a robot. A Wilcoxon signed-rank test showed that children had a statistically significant change in attitude regarding S1 (Z $=-2.265, \mathrm{p}=0.024)$ and S2 $(\mathrm{Z}=-2.207, \mathrm{p}=0.027)$.

The group that experienced the RT during the experiment did not change their attitude significantly more than the group that only experienced the HT. This is possibly due to the whole idea of having robots in the classroom being such a novel concept that it makes students somewhat cautious. After having thought about the use of a robot in the classroom by means of the questions posed in the questionnaire as well as the teacher's announcement that a robot researcher would be visiting the school, they may have grown accustomed to the idea, rendering them more positive toward robots in the post-test questionnaire, even without working with a robot.

The distributions in the two groups differed significantly regarding the change in attitude concerning two questions: 'The word "robot" means nothing to me' (Mann-Whitney $\mathrm{U}=29.000 \mathrm{n}_{H T}=9, \mathrm{n}_{R T}=12, \mathrm{p}<0.05$ two-tailed) and 'I would feel uneasy doing my schoolwork with the help of a robot' (Mann-Whitney $\mathrm{U}=26.000 \mathrm{n}_{H T}=9, \mathrm{n}_{R T}=12$, $\mathrm{p}<0.05$ two-tailed). Especially interesting is that students in the HT condition became somewhat more negative regarding this last question, while students in the robot condition became less negative. This may indicate that initial inhibitions toward potential collaborations with robots were stifled by the RT experience.

From the results, we confirm our hypothesis that students develop a more positive attitude toward an RT after the experiment, but this also holds for students not having experienced the robot. However, the significant difference in attitude change for two of the questions reveals that experience with humanoid robots can make students more positive toward collaborating with robots on schoolwork and they seem to assign conceptual meaning to robots following an interactive experience. 


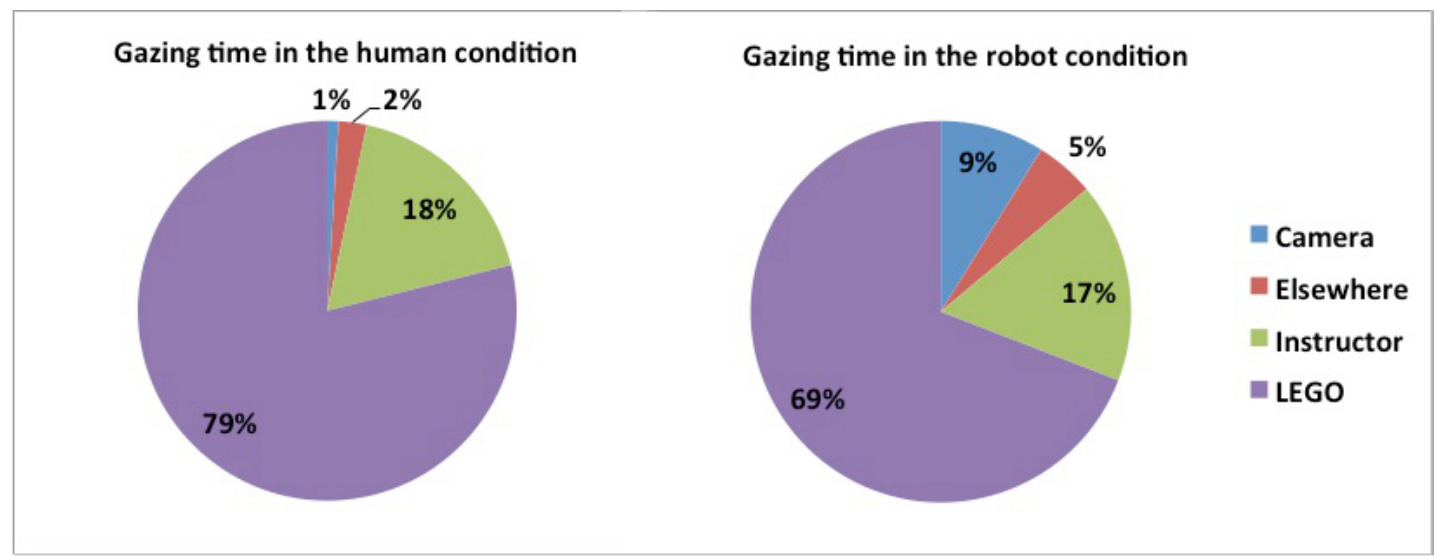

Figure 3: Percentages of time gazed in different directions for both the HT and the RT conditions

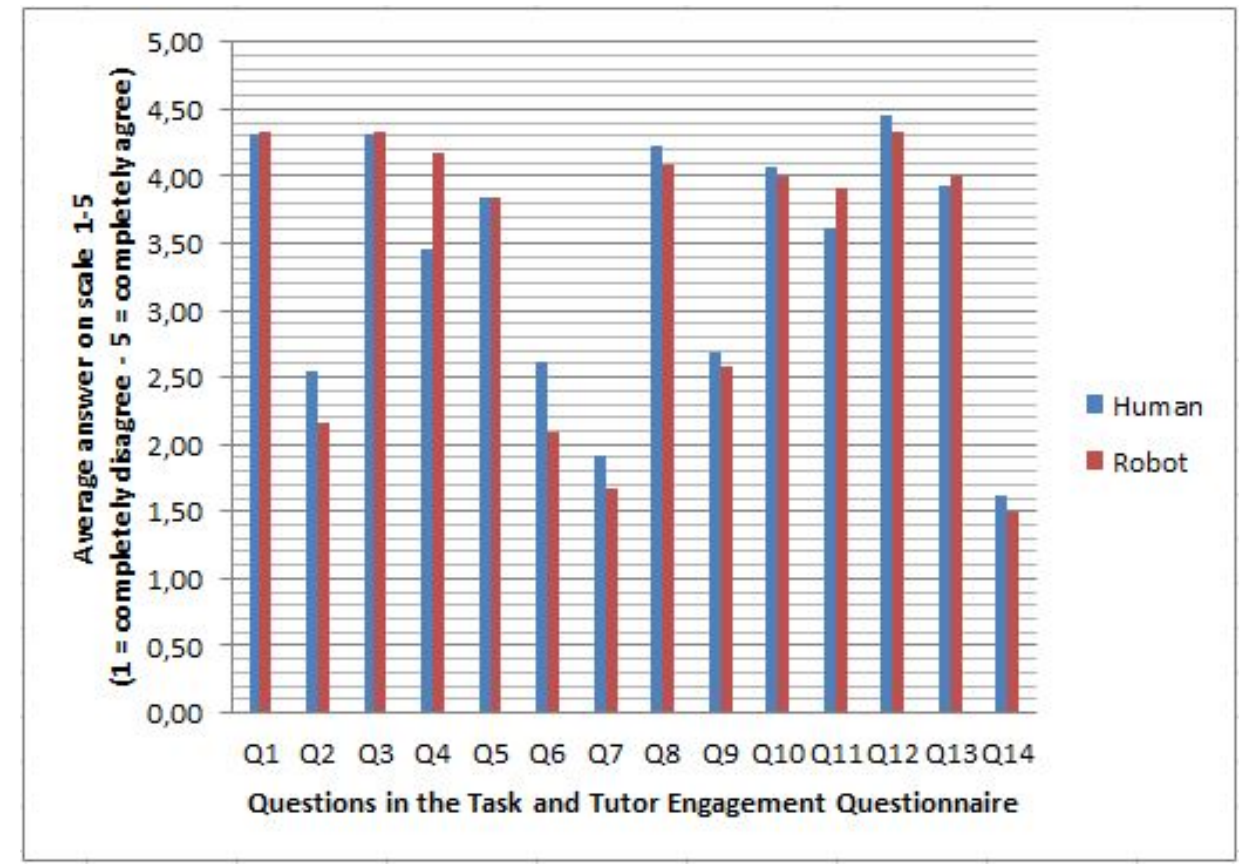

Figure 4: The average answer per question on the Task \& Tutor Engagement Questionnaire

\section{COnClusion And Future Work}

The study presented shows that students do follow basic instructions from a humanoid robot as successfully as from a human tutor. This could be related to the fact that students can be more eager to perform well with a robot than with a human. However, there are some differences: students make attempts to seek help from the human tutor, which is a nonexistent behavior in the robot tutor condition in this study. They also gaze more at the task and the instructor when working with the human, while they pay more attention to the camera when left alone with the robot.

Furthermore, all students get a more positive attitude toward robots by participating in the experiment, although the ones actually experiencing interaction with the robot show a significantly greater attitude change regarding the possibility of conducting schoolwork with a robot as well as appropriating conceptual meaning to the word "robot".
Altogether, these findings indicate the novelty for students to consider potential functions of robots within educational settings without interaction experience, since an attitude change seems to occur once interaction and/or conceptions of robots are introduced. As such, this study clearly suggests the importance of experience with humanoid robots when eliciting students' perceptions or attitudes of the same. In addition, the humanoid robot tutor displayed only minimal idle gestures. It would be of interest to explore this experimental paradigm using a humanoid robot with varying degrees of gestural movement in order to explore various effects in successful instruction following, gaze behavior and perception of the humanoid robot tutor in comparison to a human tutor.

Longer-term interaction with an autonomous humanoid robot will be necessary for students to become more accustomed to the situation. Further research is necessary 
to determine whether students keep following instructions conveyed by a robot tutor after a longer time when the potential novelty effect has been reduced.

\section{ACKNOWLEDGMENT}

We would like to thank the following people for helping with this research: Tiago Ribeiro for creating the WoZ interface, Linus Serholt for his work on the robot voice, James Wellence for assisting with technical preparations prior to the experiment, and Julia Körtge for creating the apparatus illustrations. This work was partially supported by the European Commission (EC) and was funded by the EU FP7 ICT-317923 project EMOTE (www.emote-project.eu).

\section{REFERENCES}

[1] M. Argyle and M. Cook. Gaze and Mutual Gaze. Cambridge University Press, New York, NY, USA, 1976.

[2] W. Bainbridge, J. Hart, E. Kim, and B. Scassellati. The benefits of interactions with physically present robots over video-displayed agents. International Journal of Social Robotics, 3(1):41-52, 2011.

[3] B. Barker, N. Grandgenett, and G. Nugent. Robots, gps/gis, and programming technologies: The power of "digital manipulatives" in youth extension experiences. Journal of Extension, 48(1), 2010.

[4] D. Cormier, J. Young, M. Nakane, G. Newman, and S. Durocher. Would you do as a robot commands? an obedience study for humanrobot interaction. In Proc. of the 1st International Conference on Human-Agent Interaction (iHAI13). Sapporo, Japan, 2013.

[5] J. Han. Emerging technologies: Robot assisted language learning. Language Learning \& Technology, 16(3):1-9, 2012.

[6] J. Han, M. Jo, V. Jones, and J. H. Jo. Comparative study on the educational use of home robots for children. JIPS, 4(4):159-168, 2008.

[7] W. L. Johnson, J. W. Rickel, and J. C. Lester. Animated pedagogical agents: Face-to-face interaction in interactive learning environments. International Journal of Artificial Intelligence in Education, 11:47-78, 2000.

[8] K. Jokinen, H. Furukawa, M. Nishida, and S. Yamamoto. Gaze and turn-taking behavior in casual conversational interactions. ACM Trans. Interact. Intell. Syst., 3(2):12:1-12:30, Aug. 2013.

[9] C. Kidd and C. Breazeal. Robots at home: Understanding longterm human-robot interaction. In Proc. of the IEEE/RSJ International Conference on Intelligent Robots and Systems, pages 3230-3235, 2008.

[10] C. D. Kidd and C. Breazeal. The effect of a robot on user perceptions. In Proc. IEEE/RSJ International Conference on Intelligent Robots and Systems, 2004.

[11] M.-S. Kim, J. Sur, and L. Gong. Humans and humanoid social robots in communication contexts. AI SOCIETY, 24(4):317-325, 2009.

[12] K. Lee, J.-H. Hwang, and D.-S. Kwon. A comparative study between human-human interaction and human-robot interaction. In N. Nguyen and R. Katarzyniak, editors, New Challenges in Applied Intelligence Technologies, volume 134 of Studies in Computational Intelligence, pages 3-12. Springer Berlin Heidelberg, 2008.
[13] I. Leite, C. Martinho, and A. Paiva. Social robots for long-term interaction: A survey. International Journal of Social Robotics, 5(2):291-308, 2013.

[14] D. Leyzberg, S. Spaulding, M. Toneva, and B. Scassellati. The physical presence of a robot tutor increases cognitive learning gains. In Proc. of the 34th Annual Conference of the Cognitive Science Society, 2012.

[15] M. M. Lusk and R. K. Atkinson. Animated pedagogical agents: does their degree of embodiment impact learning from static or animated worked examples? Applied Cognitive Psych., 21(6):747-764, 2007.

[16] H. A. McGregor and A. J. Elliot. Achievement goals as predictors of achievement-relevant processes prior to task engagement. Journal of Educational Psychology, 94(2):381, 2002.

[17] C. Midden and J. Ham. Using negative and positive social feedback from a robotic agent to save energy. In Proc. of the 4th International Conference on Persuasive Technology, Persuasive '09, pages 12:112:6, New York, NY, USA, 2009. ACM.

[18] O. Mubin, C. J. Stevens, S. Shahid, A. Al Mahmud, and J.-J. Dong. A review of the applicability of robots in education. Journal of Technology in Education and Learning, 1, 2013.

[19] T. Nomura, T. Suzuki, T. Kanda, and K. Kato. Measurement of negative attitudes toward robots. Interaction Studies, 7(3):437-454, 2006.

[20] A. Pereira, C. Martinho, I. Leite, and A. Paiva. icat, the chess player: The influence of embodiment in the enjoyment of a game. In Proc. of the 7th International Joint Conference on Autonomous Agents and Multiagent Systems - Volume 3, AAMAS '08, pages 1253-1256, Richland, SC, 2008. International Foundation for Autonomous Agents and Multiagent Systems.

[21] A. M. Ryan and H. Patrick. The classroom social environment and changes in adolescents' motivation and engagement during middle school. American Educational Research Journal, 38(2):437-460, 2001.

[22] M. Saerbeck, T. Schut, C. Bartneck, and M. D. Janse. Expressive robots in education: Varying the degree of social supportive behavior of a robotic tutor. In Proc. of the SIGCHI Conference on Human Factors in Computing Systems, CHI '10, pages 1613-1622, New York, NY, USA, 2010. ACM.

[23] H. N. Schifferstein and E. P. Zwartkruis-Pelgrim. Consumer-product attachment: Measurement and design implications. International Journal of Design, 2(3):1-13, 2008.

[24] N. Shin and S. Kim. Learning about, from, and with robots: Students' perspectives. In Proc. of the 16th IEEE International Symposium on Robot and Human interactive Communication (RO-MAN 2007)., pages 1040-1045, 2007.

[25] C. L. Sidner, C. Lee, C. D. Kidd, N. Lesh, and C. Rich. Explorations in engagement for humans and robots. Artificial Intelligence, 166(12): $140-164,2005$.

[26] V. Sulčič and A. Sulčič. Can online tutors improve the quality of e-learning? Issues in Informing Science \& Information Technology, 4, 2007.

[27] F. Tanaka. Robotics for supporting childhood education. In Y. Sankai, K. Suzuki, and Y. Hasegawa, editors, Cybernics: Fusion of human, machine and information systems, pages 185-195. Springer, Japan, 2014.

[28] R. Yılmaz and E. Kılıç-Çakmak. Educational interface agents as social models to influence learner achievement, attitude and retention of learning. Computers \& Education, 59(2):828 - 838, 2012. 